OPEN ACCESS

Edited by:

Awais Farid,

Hong Kong University of Science and Technology, Hong Kong SAR, China

Reviewed by:

Khayala Mammadova,

Azerbaijan National Academy of

Sciences (ANAS), Azerbaijan

Nida Gull,

Yanshan University, China

*Correspondence:

Boshi Dong

Boshi_dong@163.com

Specialty section:

This article was submitted to Organizational Psychology,

a section of the journal

Frontiers in Psychology

Received: 19 December 2021

Accepted: 04 February 2022

Published: 03 March 2022

Citation:

Dong B and Xu G (2022) An Empirical

Study on the Evaluation of Emotional Complexity in Daily Life.

Front. Psychol. 13:839133.

doi: 10.3389/fpsyg.2022.839133

\section{An Empirical Study on the Evaluation of Emotional Complexity in Daily Life}

\author{
Boshi Dong ${ }^{1 *}$ and Guangxing $X u^{2}$ \\ ${ }^{1}$ The First People's Hospital of Lianyungang, Lianyungang, China, ${ }^{2}$ School of Psychology and Cognitive Science, East China \\ Normal University, Shanghai, China
}

Emotional complexity means diversity, universality, and differentiation of individual emotions. This research consisted of two studies to demonstrate the constitution of the emotional complexity. In Study 1, the participants were asked to use 10 emotional words to record the variation of emotions over 30 days in daily life. In Study 2, the experimental materials were enriched. The participants were required to note the emotions with the Positive and Negative Affect Schedule in a 3-day holiday - all the individuals in the two studies needed to record the most important emotional event. As a result, the youth experienced complex emotions every day. Emotional complexity indicators included covariation index ( $r$ ), component index $\left(C_{p c}\right.$ and $\left.C_{\text {unshared }}\right)$, granularity index $\left(G_{e}, G_{p}\right.$, and $\left.G_{n}\right)$, and variability index $\left(V_{p}\right.$ and $\left.V_{n}\right)$. A four-factor model reflected a good model fit, with $\chi^{2} / \mathrm{df}=0.33, \mathrm{CFI}=1.00$, $\mathrm{TLI}=1.03, \mathrm{RMSEA}=0.000(0.00,0.20)$, SRMR $=0.003$, including positive differentiation, covariation of positive affect and negative affect, negative differentiation, and emotional variation. These indicators may reflect the complex experiences in everyday life. The results shed light on the emotional experience that can change greatly within 1 day and on episodes of emotional disruption resulting from an important event coupled with excessive excitement or extreme tension.

Keywords: emotional complexity, emotional events, positive affect, negative affect, psychology

\section{INTRODUCTION}

The complex emotions can be called "emotional complexity," which means diversity, universality, and differentiation of individual emotions (Ben-Ze'ev and Revhon, 2004; Grossmann et al., 2016). Wessman and Ricks (1966) used "affect complexity" to describe the complications of emotional experiences. In Handbook of Emotions, Lewis et al. (2008) further advanced the concept of emotional complexity by focusing on three main aspects, including (1) dialecticism and precision in recording emotions; (2) emotional, propositional knowledge in different situations; and (3) complexity of self-characterizations.

There are also two kinds of perspectives that define emotions' nature (Hay and Diehl, 2011; Grossmann et al., 2016). The instinctive orientation considers a series of emotions as genetic and fixed categories (Roseman, 1984; Lindquist et al., 2006). Barrett and Bliss-Moreau (2009) suggests that when more than one kind of emotion is triggered at a particular moment, or when people accurately describe the variety in their emotional experience, they will self-report the emotional complexity. Then people gain propositional knowledge about emotions when they learn to associate a given emotion with the environment. Therefore, the emotional strategy 
is a perceptual strategy, which means one can get the coding strategies at birth and gradually adapt to different environments (Grossmann et al., 2016). Self-characterization of emotional complexity is reflected in individuals' ability to recognize their experiences as complex (Berrios, 2019).

Another way to define the nature of emotion uses a "psychological construction" orientation (Russell, 2003, 2017; Barrett, 2004; Feldman et al., 2007). It holds that emotional complexity is intrinsic to the emotional experiences of neurobiological and psychological systems. A discrete emotion emerges in consciousness when a more basic core affect is automatic and implicit. The core effect is a constantly changing state with valence and arousal-based properties (Russell, 2003; Barrett, 2004). Current studies use the "emotional conception system" to classify core effects, such as anger, sadness, and fear. This term refers to the knowledge about specific emotions that people understand to clarify effectively and automatically attribute core affect to a certain state that is efficient and conceptual when inner feelings of the body and the external information from the environment are bound together for a moment in time. Categorizing emotions transforms core affect into an intentional state, allowing individuals to explore what caused the emotional change, what to do next, and how to communicate to others effectively and efficiently (Barrett, 2004). Therefore, emotional complexity is the direct result of the emotional conception system.

In addition, emotional complexity includes at least two different simultaneous or sequential emotions simultaneously. A sequential or continuous state is a rapid change in two or more emotional states, such as a pure emotion appearing first and then being quickly replaced by another emotion. Conversely, a simultaneous complex emotion' responds to stimuli with two or more opposite emotions' (Larsen et al., 2001). Rothman et al. (2017) point out that simultaneous states include mixed emotions and emotional ambivalence, and sequential states include affective transition and emotional inconsistencies. The definitions and differences in these states stem from trait factors, including effective spin, affective variability, emotional inertia, and emotional complexity (Kang and Shaver, 2004). Therefore, some current studies measure emotional complexity by the level of emotional awareness (Ben-Ze'ev and Revhon, 2004; Berrios, 2019). Some researchers stated mixed emotions as indicators of emotional complexity (Trudel-Fitzgerald et al., 2017).

Emotional complexity is multiply defined, often regarding the co-occurrence and differentiation of positive and negative emotions as the leading indices (Hay and Diehl, 2011; Grühn et al., 2013; Larsen, 2017). The co-occurrence index reflects the two opposite valences in emotions (e.g., happiness and sadness) simultaneously (Ong and Bergeman, 2004). In contrast, differentiation of positive and negative emotions, named granularity index, reflects the characteristic distinction among emotions that one makes (Ong and Bergeman, 2004).

Although most emotional experiences can be represented by positive and negative dimensions (Barrett, 1998; Charles et al., 2017; Butler et al., 2018), the degree to which adults distinguish emotions is different (Ong et al., 2017). Barrett (2004) argued that people first experience core affect, which they then categorize as a particular emotion (e.g., anger, frustration, etc.). It is essential to assess emotional complexity through the co-occurrence and granularity index. Barrett (2004) studies shows that self-reports of emotions and emotion labels used in conception reflect more forms in the emotional words when differentiating; and they also reflect the differences in adults' phenomenological experience.

Zautra et al. (2005) proposed Dynamic Model of Affect (DMA) to explore individuals' differences of emotions in time. DMA asserts that it is uncertain to descript emotional experiences, and pressure in situations will increase the uncertainty. Information processing requires cognitive strategies and becomes more complex when complicated emotional experiences. Consequently, DMA suggests that emotions will polarize in a high-pressure environment. For example, a high level of negative emotion is often accompanied by a low level of positive emotion. DMA aims to assess emotional states without ignoring one's stress level, which means that the relationship between positive and negative emotion may change with information processing (such as cognitive and emotional processing methods). Moreover, it is important to construct at least two dimensions to generally classify emotions, one to assess negative emotion with the accompanying motivation level, and the other to assess the level of positive emotion with the accompanying processing (Ramsey et al., 2016).

One groundbreaking approach uses "micro longitudinal" techniques to assess emotions and intense events at multiple points (Kreibig and Gross, 2017; Ong et al., 2017). This method involves noting the correlation between positive and negative emotions at each time point and observing what happens to this association when pressure or other destructive events occur in daily life. Furthermore, people can determine whether a stressful event was greater in magnitude or occurred with greater frequency than their average experience by repeatedly measuring these co-occurring experiences. To obtain the deviations from the mean values, the independent variables are person-centered. Person-centered negative affect (NA) can be used as a predictor in a typical model to estimate its association with positive affect (PA) over time in the context of a linear mixed model. In this case, choosing which valence should be modeled as the independent versus dependent variable is random but may be guided by particular research questions.

This study aimed to explain daily emotional experience traits in the youth and considered emotional complexity as a state (the co-occurrence of positive and negative emotions). To investigate the simultaneous and sequential emotional states, we set up two studies to explore the indicators to describe the complex emotions in everyday life. Through the studies, we could find the emotional complexity index and investigate the relationship among the indicators. And also, we would examine the emotional states of the daily experiences in a different environment.

\section{MATERIALS AND METHODS}

Study 1 contained 61 participants without psychiatric disorders from a normal university in East China who were initially 
invited to join in the study. All the participants were drawn randomly from the university. Fifteen participants who completed less than half of the planned measurement points (once a day for 30 days) were excluded. The final sample included solely Han Chinese, ranging in age from 18 to 24 years old; most of the sample were women (53\%). Participants signed an informed consent document before recording the emotions. After 30 day-dairy, participants were rewarded for a gift.

Study 2 consisted of 46 participants recruited from a normal university in East China without psychiatric disorders. This sample included solely Han Chinese, ranging in age from 18 to 24 years; most of the sample were women $(70 \%)$. Before initiating the experiment, participants read and signed an informed consent document. After completing the experiment, participants were rewarded with a gift.

\section{Procedures}

Study 1: Using an online questionnaire, participants were asked how often ( 1 =none, 7 =always $)$ they felt each emotion in the last 24h (Ready et al., 2007, 2008). The emotions consisted of positive and negative items, including happy, joyful, pleased, enjoyment/fun, content, depressed, unhappy, frustrated, angry/ hostile, and worried/anxious. Participants completed the online questionnaire about their emotions and experiences for 30 consecutive evenings.

Study 2: Data were collected during the three-day New Year holiday using an affect measure with more independent PA and NA scales than the instrument used in study 1 . Demographic information was entered online, and the Positive and Negative Affect Schedule (PANAS) was completed online every day over the 3-day holiday (Watson et al., 1988). The PANAS exhibited strong internal consistency (PA $\alpha=0.90$; NA $\alpha=0.87$ ) and 2-month test-retest reliability (PA $r=0.47$; NA $r=0.39)$.

\section{Data Analysis}

Consistent with existing research, this study used principal components analysis (PCA) to estimate the data of emotional records in study 1 and study 2 (Carstensen et al., 2000; Ong and Bergeman, 2004; Grühn et al., 2013). The covariation score was defined as the within-person correlation between daily reports of positive and negative $\left(r_{\mathrm{pn}}\right)$, where a coefficient close to 0 indicated greater complexity among emotions. The component scores were obtained by within-person principal components factor analysis. They were taken from the number of factors with eigenvalues greater than 1 , where higher values indicated higher levels of emotional complexity. The other component score was calculated by subtracting the variance accounted for in the first extracted principal component factor from $100 \%\left(C_{\text {unshared }}\right)$.

Granularity scores were computed from each participant's inter-class correlation coefficient (ICC) index subtracted from one (McGraw and Wong, 1996; Grühn et al., 2013). Low granularity scores reflect high consistency among emotion reports and different forms of emotion expressions. Therefore, we calculated three items: emotion granularity $\left(G_{\mathrm{e}}\right)$, positive emotions $\left(G_{\mathrm{p}}\right)$, and negative emotions $\left(G_{\mathrm{n}}\right)$. The variability scores consisted of two items calculated from the standard deviation of the positive and negative emotions from the experience-sampling data, including positive affect $\left(V_{\mathrm{p}}\right)$ and negative affect $\left(V_{\mathrm{n}}\right)$.

Statistical analysis was conducted with SPSS 25.0 and Mplus 7.4. The data from participants who reported the same score continuously in the records were excluded from the analysis. The participants need to report the most important emotional events in the two studies. The emotional reaction to the event could be strong and last all day. Then the narratives were coded for numbers of emotional units (i.e., sentence/phrase that conveyed a complete thought). Narratives also were coded for thematic content: Physical health; Interpersonal social; Recreation; Education; and Natural environment. The discrepancies of all coding were resolved via discussion.

\section{RESULTS}

Table 1 showed that the daily life PA and NA scores were close to the emotional scores in holiday. For instance, PA decreased, and NA scores were lower than baseline because of the environment change and the effect of time. The effects also involved emotional vocabulary and cultural background (Bagozzi et al., 1999). The vocabulary selected in Study 2(a) reflected the core affect, and the PANAS responses in Study 2(b) reflected the emotional survey, especially regarding the polarization of emotions. However, in Study 1, the emotional adjectives were based on the Chinese context for special emotions. At the same time, Study 2 resulted in different environments, consistent with the conclusion in Study 1.

The within-person correlation between PA and NA in Study 1 was moderately negative (see Table 1 ). However, in Study 2, there was no correlation between PA and NA (see Table 2). The emotional frequency of individuals' experiences showed the same trend in the two experiments. Participants reported more positive emotions than negative emotions in daily life and over the holiday. The proportion of PA and NA occurring simultaneously was the highest in the two experiments. The no-emotion proportion was only $0.47 \%$ among the four emotional reports, especially in Study 2. And the participants did not report any emotions on holiday. It was noteworthy that the total proportion of no-emotion and NA reports accounted for only $1.44 \%$ of the variance, whereas PA and

TABLE 1 | Descriptive statistics of the emotional records in study 1.

\begin{tabular}{lcc}
\hline & $\boldsymbol{M} \pm \mathbf{S D}$ & Frequency (\%) \\
\hline PA & $4.25 \pm 1.74$ & 30.82 \\
NA & $2.09 \pm 1.25$ & 2.90 \\
PA and NA & & 65.80 \\
None & & 0.47 \\
Correlation & $-0.41 \pm 0.28$ &
\end{tabular}

PA, positive affect; NA, negative affect; None: "1" means no such emotion in the emotional report. 
TABLE 2 | Descriptive statistics of the emotional records in study 2.

\begin{tabular}{lcc}
\hline & $\boldsymbol{M} \pm \mathbf{S D}$ & Frequency (\%) \\
\hline PA & $2.91 \pm 0.82$ & 5.04 \\
NA & $1.99 \pm 0.76$ & 1.44 \\
PA and NA & & 93.43 \\
None & & 0 \\
Correlation & $0.01 \pm 0.8$ & \\
\hline
\end{tabular}

$P A$, positive affect; NA, negative affect; None: "1" means no such emotion in the emotional report.

NA accounted for $93.43 \%$ (see Table 2 and Figure 1). The results indicated that the youth's emotional experience was richer and more diverse during the holidays.

Table 3 showed the indicators of emotional complexity, including the covariation index $(\mathrm{r})$; component index $\left(C_{\mathrm{pc}}\right.$ and $\left.C_{\text {unshared }}\right)$; granularity index $\left(G_{\mathrm{e}}, G_{\mathrm{p}}\right.$, and $\left.G_{\mathrm{n}}\right)$ and variability index $\left(V_{\mathrm{p}}\right.$ and $\left.V_{\mathrm{n}}\right)$. There were different degrees of correlation between the indicators. The covariation score had a significant correlation with the component and granularity scores, except for Cunshared and the variability scores. However, there was a lack of component indicators in Study 2 (see Table 4). The results also showed that $G_{\mathrm{n}}$ was positively correlated with $V_{\mathrm{p}}$, which differed from the results in Study 2 (Table 5).

To further identify the predictive effects of the indicators for emotional complexity, we performed Exploratory Factor Analysis and Confirmatory Factor Analysis (Hopwood and Donnellan, 2010; Khalil et al., 2021; Khawaja et al., 2021). A four-factor model reflected a good model fit, with $\chi^{2} / \mathrm{df}=0.33$, $\mathrm{CFI}=1.00, \mathrm{TLI}=1.03, \mathrm{RMSEA}=0.000(0.00,0.20), \mathrm{SRMR}=0.003$. The results showed that some indicators were related to multiple factors (see Table 4 and Figure 2).

\section{Important Emotional Events}

The period from 18 to 24 years old in China is filled with education; just as the participants' records of important events, the most frequent emotional event was "courses." Because the participants were mainly selected from normal universities, life in the campus was closely related to their daily experiences. However, the courses included calligraphy, piano, painting, dancing, and professional lessons. As a result, the participants could feel more complex emotions, such as depression, excitement, sadness, anxiety, calm, happiness, etc. The narrative could also be described by the words "busy and productive" (Butler et al., 2018; Sarfraz et al., 2019; Gull et al., 2021; Xiaolong et al., 2021), not only because most of the participants were firstyear students but also the heavy professional courses and colorful activities occupied the campus time with no leisure. Then life was dominated by complex emotions.

In the participants' logs, the negative emotions, such as fatigue, irritability, and discomfort, were often associated with physical health, including "feeling cold," "illness," and "discomfort" (see Table 6). While emotions related to "homesickness" were depression and anxiety. "Examination" was also an important cause of negative emotions, such as tension and fatigue before the exam and concerns about performance. The other topics that mostly resulted in the mood swings were weather, diner, and journey. The events for the first time in individuals' experiences affected the occurrence and development of emotions, including intimate relationships and part-time work.

At the same time, attention to social events made participants experience complex emotions. Typical events included: "I was satisfied with the state of learning, but I felt depressed and distressed for the victims in the nanny;" "Hell is empty and demons are on earth. The arson case that has been concerned has not been concluded so far. However, the other cases ended up with nothing definite. In fact, the case named three colors have been disclosed for 15 years, but it was not until these two days those social concerns began."

\section{DISCUSSION}

The PA and NA scores in Study 2 were lower than those in Study 1; when the emotional record was short and away from the familiar environment, the PA and NA scores changed substantially. This may be explained by errors in statistical analysis or the relationships among emotional complexity, time, and environmental cues. Regarding the correlation between PA and NA, the 30-day records showed significance, but the 3-day records were insignificant, and the related coefficient was close to zero. Results in Study 1 demonstrated that while some people experienced many different emotions, their positive and negative emotions tended to be opposite (Reich et al., 2003; Grühn et al., 2013; Scott et al., 2014). There was no correlation between PA and NA in Study 1, indicating that these emotional records were discrete, or that individuals experienced a deeper complexity of emotions when separated from their usual environment. Individuals were more likely to experience the excitement on graduation and leisure days, which would reduce pressure and tension (Larsen et al., 2001).

The results of emotional frequency were consistent with previous studies (Grühn et al., 2013), but the proportion of no-emotion in Study 1 was 0 , and PA and NA accounted for more than $90 \%$ of the variance. This extreme result suggests that when individuals interrupted their habits, their ability to distinguish emotions decreased, and some experienced emotional ups and downs during the exceptional time, which might affect the occurrence of PA and NA. In other words, richer emotional events resulted in more complex emotions. This suggests the co-activation of PA and NA; when the emotional valence was different, a complex emotional experience occurred.

The emotional complexity indicators in studies were inconsistent because Study 2 only collected data for 3 days, resulting in a smaller amount of data, which could not be analyzed with factor analysis. This made it difficult to get the component indicators of $C_{\mathrm{pc}}$ and $C_{\text {unshared }}$. There was no fluctuation indicator in present studies, which differed from the results of previous studies (Grühn et al., 2013). The potential factors obtained by confirmatory factor analysis were consistent with the former study. Still, the items included in the factors were different because the emotions in studies were based on days, and the data in the formal study were based on time 


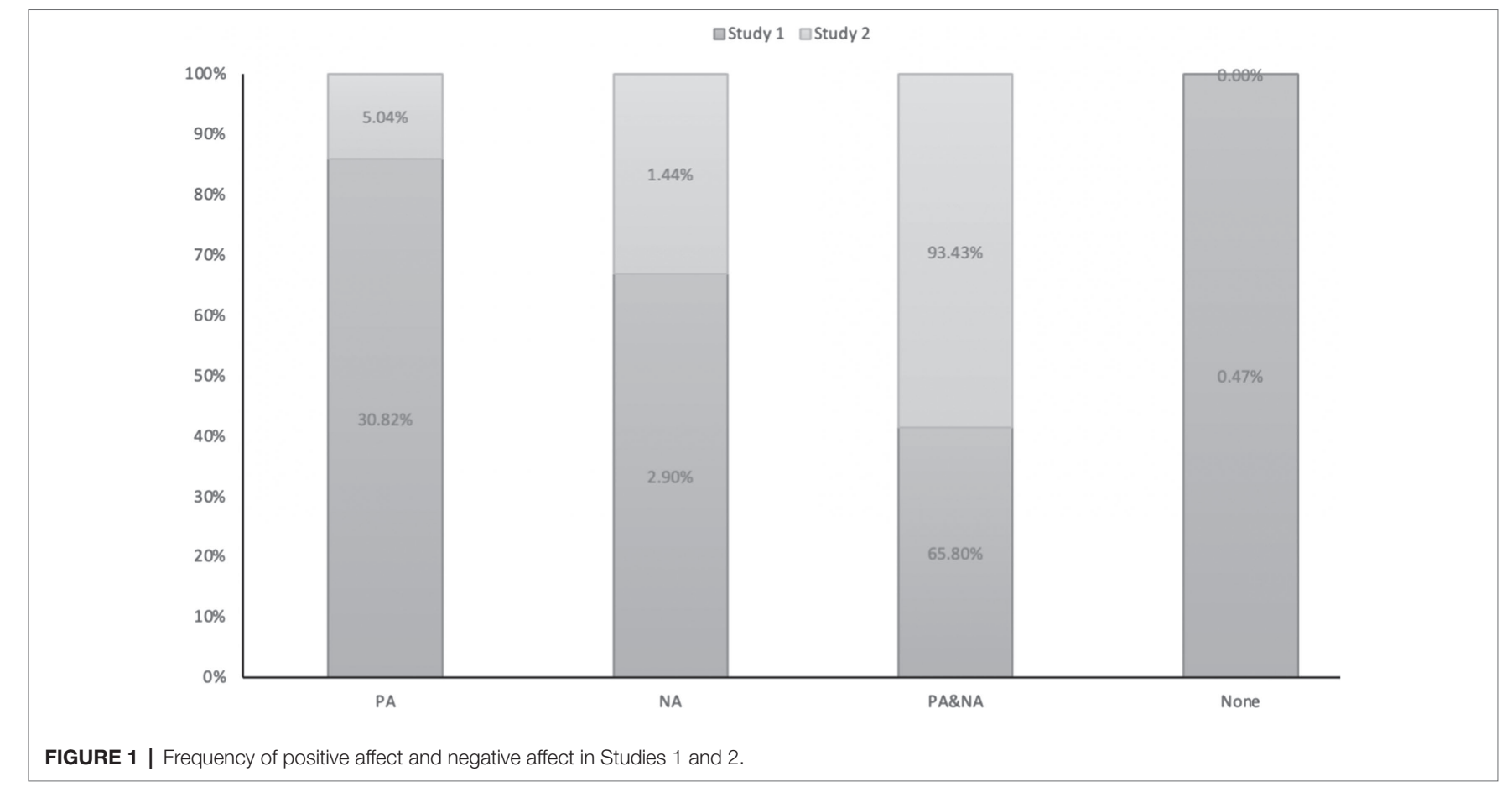

TABLE 3 | Associations between indicators of emotional complexity in Study 1.

\begin{tabular}{|c|c|c|c|c|c|c|c|c|}
\hline & $r_{\mathrm{pn}}$ & $C_{\mathrm{PC}}$ & $\boldsymbol{C}_{\text {unshared }}$ & $G_{e}$ & $G_{p}$ & $G_{n}$ & $V_{p}$ & $V_{\mathrm{n}}$ \\
\hline$r_{\mathrm{pn}}$ & 1 & & & & & & & \\
\hline$C_{P C}$ & $0.59 * *$ & 1 & & & & & & \\
\hline$C_{\text {unshared }}$ & 0.16 & 0.11 & 1 & & & & & \\
\hline$G_{e}$ & $-0.74 * *$ & $-0.43^{* *}$ & 0.10 & 1 & & & & \\
\hline$G_{p}$ & 0.33* & $0.42 * *$ & $0.59 * *$ & 0.04 & 1 & & & \\
\hline$G_{n}$ & $0.60 * *$ & 0.66** & $0.44 * *$ & -0.28 & $0.48 * *$ & 1 & & \\
\hline$V_{p}$ & -0.12 & -0.26 & $-0.38 *$ & -0.15 & $-0.65 * *$ & -0.07 & 1 & \\
\hline$V_{\mathrm{n}}$ & -0.29 & $-0.53 * *$ & $-0.31 *$ & 0.13 & $-0.48 * *$ & $-0.52 * *$ & $0.54 * *$ & 1 \\
\hline
\end{tabular}

$r_{p n}$, covariation score; $C_{p c}$ and $C_{\text {unshared, }}$ component score; $G_{e}, G_{p}$, and $G_{n}$, granularity score; $V_{p}$ and $V_{n}$, variability score. $* p<0.05$ and $* * p<0.01$.

TABLE 4 | Associations between indicators of emotional complexity in Study 2.

\begin{tabular}{cccccc}
\hline & $\boldsymbol{r}_{\mathrm{pn}}$ & $\boldsymbol{G}_{\mathbf{e}}$ & $\boldsymbol{G}_{\mathrm{p}}$ & $\boldsymbol{G}_{\mathbf{n}}$ & $\boldsymbol{V}_{\mathbf{p}}$ \\
\hline$r_{\mathrm{pn}}$ & 1 & & & & \\
$G_{\mathrm{e}}$ & $-0.445^{* *}$ & 1 & & & \\
$G_{\mathrm{p}}$ & 0.096 & 0.183 & 1 & & \\
$G_{\mathrm{n}}$ & -0.122 & -0.035 & -0.038 & 1 & 1 \\
$V_{\mathrm{p}}$ & 0.113 & -0.124 & $-0.507^{* *}$ & $0.418^{*}$ & \\
$V_{\mathrm{n}}$ & 0.035 & -0.127 & $-0.381^{* *}$ & $-0.517^{* *}$ & 0.116 \\
\hline
\end{tabular}

${ }^{\star}$ Correlation is significant at the 0.05 level $(p<0.05) .{ }^{\star *}$ Correlation is significant at the 0.01 level $(p<0.01)$.

points. Different approaches to data processing also led to differences between previous results and present consequences.

The covariation index represented the overall trend of change, where PA and NA were moderately negatively correlated. The low covariation between PA and NA suggested a bipolar tendency of emotions, suggesting that individuals may experience a higher degree of emotional complexity. The high correlation between
PA and NA indicated that individuals could not recognize emotional states. The low correlation indicated the relative independence of PA and NA, and it may suggest that people experience complex emotions more easily (Palgi et al., 2014). The covariation between PA and NA was more negative under non-stress conditions where the correlation coefficient was almost zero (Scott et al., 2014). The component index resulted 
TABLE 5 | Latent factors.

\begin{tabular}{lrrrl}
\hline & PD & COV & ND & EV \\
\hline$r$ & 0.70 & 0.39 & & \\
$C_{P C}$ & 0.94 & & & 0.29 \\
$C_{\text {unshared }}$ & -0.95 & & & \\
$G_{e}$ & & 0.97 & & \\
$G_{p}$ & -0.99 & & 0.39 & \\
$G_{n}$ & & & -0.77 & 0.66 \\
$V_{p}$ & 0.90 & & 0.04 & 0.53 \\
$V_{n}$ & & -0.44 & 0.577 & \\
\hline
\end{tabular}

$P D$, positive differentiation; COV, covariation of $P A$ and $N A ; N D$, negative differentiation; $E V$, emotional variation.

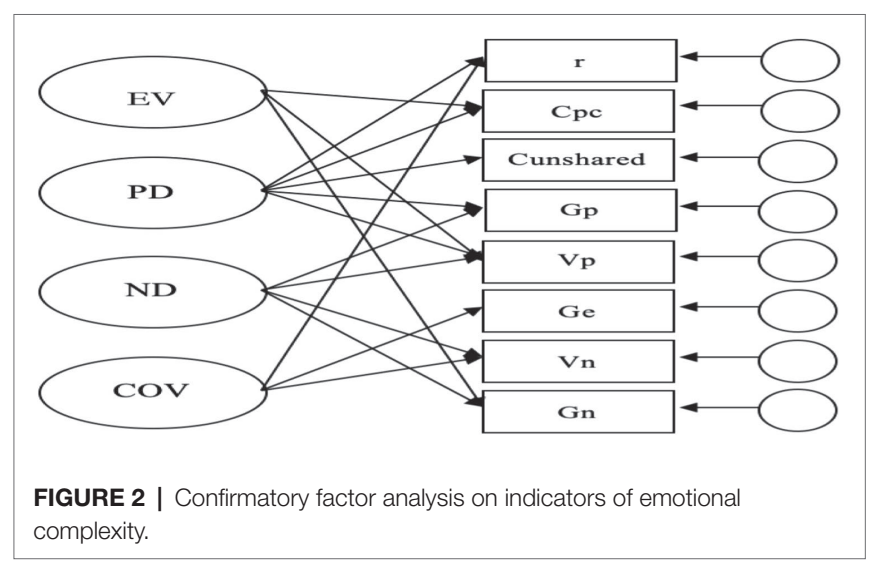

from factor analysis, which can only calculate an index when the data reach a certain statistic. For example, Study 2 was limited to only 3 days, and insufficient data were obtained to conduct a factor analysis.

Granularity indicators were quantified by the individual's ability to emphasize the value and arousal of core affect when reporting experiences. High scores were derived from using different vocabulary to describe diverse experiences, such as anger and sadness, which were completely different emotional experiences. Participants with low emotional granularity used the same series of words to describe the experience as participants with high emotional granularity. Still, the low granularity participants used the words to express basic emotional states. They used "anger," "sadness," or "fear" to describe "unhappy;" and "excited," "delight," or "cool" to describe "happy." There were also a few who reported "excited" and "nervous" as similar experiences, and they replaced these words with similar vocabulary in arousal. Low granularity reflected a high degree of consistency in the emotional reports, whereas high granularity indicated that individuals used different emotional vocabulary to describe their experiences. Participants with high granularity reported different emotions over a given time, suggesting a superior ability to distinguish emotions. In contrast, participants with low granularity only reported one kind of emotion.

The variability indicator showed a tendency toward emotions, but there was no significant correlation with mental health, happiness, or personality (Grühn et al., 2013). The results also
TABLE 6 | Frequency of important emotional events.

\begin{tabular}{|c|c|c|c|}
\hline \multirow{2}{*}{\multicolumn{2}{|c|}{ Important emotional events }} & \multicolumn{2}{|c|}{ Frequency } \\
\hline & & Positive affect & Negative affect \\
\hline \multirow[t]{7}{*}{ Life in campus } & Courses & 119 & 69 \\
\hline & $\begin{array}{l}\text { Homework (calligraphy, } \\
\text { piano, painting, } \\
\text { dancing, etc.) }\end{array}$ & 96 & 85 \\
\hline & Examination & 45 & 62 \\
\hline & $\begin{array}{l}\text { Activities on campus } \\
\text { (meetings, } \\
\text { competitions, dinners, } \\
\text { etc.) }\end{array}$ & 78 & 69 \\
\hline & Intimate relationship & 27 & 25 \\
\hline & Dormitory relationships & 10 & 30 \\
\hline & $\begin{array}{l}\text { Daily life (getting up, } \\
\text { sleeping, eating, etc.) }\end{array}$ & 55 & 60 \\
\hline $\begin{array}{l}\text { Natural } \\
\text { environment }\end{array}$ & $\begin{array}{l}\text { Weather (sunny, rainy, } \\
\text { windy, etc.) }\end{array}$ & 15 & 20 \\
\hline \multirow[t]{2}{*}{ Personal factors } & $\begin{array}{l}\text { Physical health (feeling } \\
\text { cold, illness, discomfort, } \\
\text { etc.) }\end{array}$ & 0 & 32 \\
\hline & Introspection & 40 & 46 \\
\hline \multirow[t]{3}{*}{ Entertainment } & $\begin{array}{l}\text { Online games and } \\
\text { mobile games }\end{array}$ & 34 & 30 \\
\hline & $\begin{array}{l}\text { Sports (running, } \\
\text { basketball, volleyball, } \\
\text { etc.) }\end{array}$ & 58 & 16 \\
\hline & $\begin{array}{l}\text { Communication with } \\
\text { family members }\end{array}$ & 76 & 12 \\
\hline \multirow{2}{*}{$\begin{array}{l}\text { Extracurricular } \\
\text { activities }\end{array}$} & Practice, part-time work & 20 & 20 \\
\hline & (Journey, home) & 89 & 78 \\
\hline
\end{tabular}

demonstrated that the covariation index, component indicators, and variability indicators were not related to mental health or alexithymia. This might be explained by a cognitive development perspective (Labouvie-Vief et al., 2007), where emotions involve both complexity and different levels of integration, suggesting a meaningful combination of experiences concerning self and others. In this view, it may be necessary to clarify whether complexity was incorporated into adaptive adjustment. Labouvie-Vief and Medler (2002) conducted a series of surveys and found that integrated individuals were highly complex and integrated. Complex individuals had high complexity and low integration. The complexity of defensive individuals was low, and the degree of integration was high, while the dysfunctional individuals were low in complexity and integration. These results suggest that complexity could not reflect the function of adaptation, but the complexity is related to dysfunction.

\section{Limitations and Future Research}

Emotional complexity is an intricate conception. The purpose of the present studies was to evaluate the youth's emotional complexity and explore different realistic and theoretical issues. However, there were some limitations that the data pertaining to the same event can point to very different implications about emotional complexity. For example, depending on whether data were gathered via a rating task or via diary data averaged 
over days. Therefore, the definition and measurement of emotional complexity is important to adult development because there

is a risk of mixed and confusing signals from emotional complexity research when different metrics are compared.

Measurement issues also matter if one is curious about potential correlates of emotional complexity. Emotion measures with subscales or items that are relatively independent may be ideal. Another issue to tackle is whether particular emotional complexity metrics have similar meanings. Future researchers should pay close attention to methodology to address these concerns. This study presented data from diary studies in daily life. It tested several theory-based predictions using numerous conceptualizations of complex emotions. Given the novel conclusions based on the data and the lack of long-term follow-up, it is impossible to determine whether co-occurrence is adaptive for later outcomes. Future studies should consider these procedures and findings within a longitudinal framework. In addition, small convenience samples were used.

\section{CONCLUSION}

In summary, the current studies provide a systematic examination of the experience of complex emotions in daily life among the youth. The results shed light on the emotional experience that can change greatly within 1 day and on episodes of emotional disruption resulting from an important event coupled with

\section{REFERENCES}

Bagozzi, R. P., Wong, N., and Yi, Y. (1999). The role of culture and gender in the relationship between positive and negative affect. Cogn. Emot. 13, 641-672. doi: 10.1080/026999399379023

Barrett, L. F. (1998). Discrete emotions or dimensions? The role of valence focus and arousal focus. Cogn. Emot. 12, 579-599. doi: 10.1080/026999398379574

Barrett, L. F. (2004). Feelings or words? Understanding the content in selfreport ratings of experienced emotion. J. Pers. Soc. Psychol. 87, 266-281. doi: 10.1037/0022-3514.87.2.266

Barrett, L. F., and Bliss-Moreau, E. (2009). Affect as a psychological primitive. Adv. Exp. Soc. Psychol. 41, 167-218. doi: 10.1016/S0065-2601(08)00404-8

Ben-Ze'ev, A., and Revhon, N. (2004). Emotional complexity in everyday life. Soc. Sci. Inf. 43, 581-589. doi: 10.1177/0539018404047704

Berrios, R. (2019). What is complex/emotional about emotional complexity? Front. Psychol. 10:1606. doi: 10.3389/fpsyg.2019.01606

Butler, R. M., Boden, M. T., Olino, T. M., Morrison, A. S., Goldin, P. R., Gross, J. J., et al. (2018). Emotional clarity and attention to emotions in cognitive behavioral group therapy and mindfulness-based stress reduction for social anxiety disorder. J. Anxiety Disord. 55, 31-38. doi: 10.1016/j. janxdis.2018.03.003

Carstensen, L. L., Pasupathi, M., Mayr, U., and Nesselroade, J. R. (2000). Emotional experience in everyday life across the adult life span. J. Pers. Soc. Psychol. 79:644. doi: 10.1037/0022-3514.79.4.644

Charles, S. T., Piazza, J. R., and Urban, E. J. (2017). Mixed emotions across adulthood: when, where, and why? Curr. Opin. Behav. Sci. 15, 58-61. doi: 10.1016/j.cobeha.2017.05.007

Feldman, L., Mesquita, B., Ochsner, K. N., and Gross, J. J. (2007). The experience of emotion. Annu. Rev. Psychol. 58, 373-403. doi: 10.1146/annurev. psych.58.110405.085709

Grossmann, I., Huynh, A. C., and Ellsworth, P. C. (2016). Emotional complexity: clarifying definitions and cultural correlates. J. Pers. Soc. Psychol. 111:895. doi: $10.1037 /$ pspp0000084 excessive excitement or extreme tension. These results are fully consistent with the definition of emotional complexity, which asserts that people can experience two or more emotions simultaneously or sequentially. The results also fit well with the primary indicators of emotional complexity, PA and NA, which coexist in the individual's experience where pure emotion rarely appears.

\section{DATA AVAILABILITY STATEMENT}

The raw data supporting the conclusions of this article will be made available by the authors, without undue reservation.

\section{ETHICS STATEMENT}

Ethical review and approval was not required for the study on human participants in accordance with the local legislation and institutional requirements. The patients/participants provided their written informed consent to participate in this study.

\section{AUTHOR CONTRIBUTIONS}

All authors listed have made a substantial, direct, and intellectual contribution to the work and approved it for publication.

Grühn, D., Lumley, M. A., Diehl, M., and Labouvie-Vief, G. (2013). Timebased indicators of emotional complexity: interrelations and correlates. Emotion 13:226. doi: 10.1037/a0030363

Gull, N., Song, Z., Shi, R., Asghar, M., Rafique, M. A., and Liu, Y. (2021). Paternalistic leadership, polychronicity, and life satisfaction of nurses: the role of work-family conflict and family-work conflict in public sector hospitals. Front. Psychol. 12:648332. doi: 10.3389/fpsyg.2021.648332

Hay, E. L., and Diehl, M. (2011). Emotion complexity and emotion regulation across adulthood. Eur. J. Ageing 8, 157-168. doi: 10.1007/s10433-011-0191-7

Hopwood, C. J., and Donnellan, M. B. (2010). How should the internal structure of personality inventories be evaluated? Personal. Soc. Psychol. Rev. 14, 332-346. doi: 10.1177/1088868310361240

Kang, S., and Shaver, P. R. (2004). Individual differences in emotional complexity: their psychological implications. J. Pers. 72, 687-726. doi: 10.1111/j.0022-3506.2004.00277.x

Khalil, M., Khawaja, K. F., and Sarfraz, M. (2021). The adoption of blockchain technology in the financial sector during the era of fourth industrial revolution: a moderated mediated model. Qual. Quant., 1-18. doi: 10.1007/ s11135-021-01229-0

Khawaja, K. F., Sarfraz, M., Rashid, M., and Rashid, M. (2021). How is COVID-19 pandemic causing employee withdrawal behavior in the hospitality industry? An empirical investigation. J. Hosp. Tour. Insights. doi:10.1108/ JHTI-01-2021-0002 [Epub ahead of print].

Kreibig, S. D., and Gross, J. J. (2017). Understanding mixed emotions: paradigms and measures. Curr. Opin. Behav. Sci. 15, 62-71. doi: 10.1016/j. cobeha.2017.05.016

Labouvie-Vief, G., Diehl, M., Jain, E., and Zhang, F. (2007). Six-year change in affect optimization and affect complexity across the adult life span: a further examination. Psychol. Aging 22:738. doi: 10.1037/0882-7974.22.4.738

Labouvie-Vief, G., and Medler, M. (2002). Affect optimization and affect complexity: modes and styles of regulation in adulthood. Psychol. Aging 17:571. doi: $10.1037 / 0882-7974.17 .4 .571$

Larsen, J. T. (2017). Introduction to the special section on mixed emotions. Emot. Rev. 9, 97-98. doi: 10.1177/1754073916672523 
Larsen, J. T., McGraw, A. P., and Cacioppo, J. T. (2001). Can people feel happy and sad at the same time? J. Pers. Soc. Psychol. 81, 684. doi: 10.1037/0022-3514.81.4.684

Lewis, M., Haviland-Jones, J. M., and Barrett, L. F. (2008). Handbook of Emotions. 3rd Edn. New York, NY: Guilford Press.

Lindquist, K. A., Barrett, L. F., Bliss-Moreau, E., and Russell, J. A. (2006). Language and the perception of emotion. Emotion 6:125. doi: 10.1037/1528-3542.6.1.125

McGraw, K. O., and Wong, S. P. (1996). Forming inferences about some intraclass correlation coefficients. Psychol. Methods 1:30. doi: 10.1037/1082-989X.1.1.30

Ong, A. D., and Bergeman, C. S. (2004). The complexity of emotions in later life. J. Gerontol. Ser. B Psychol. Sci. Soc. Sci. 59, P117-P122. doi: 10.1093/ geronb/59.3.P117

Ong, A. D., Zautra, A. J., and Finan, P. H. (2017). Inter-and intra-individual variation in emotional complexity: methodological considerations and theoretical implications. Curr. Opin. Behav. Sci. 15, 22-26. doi: 10.1016/j. cobeha.2017.05.018

Palgi, Y., Shrira, A., Ben-Ezra, M., Spalter, T., Kavé, G., and Shmotkin, D. (2014). Age-related and death-related differences in emotional complexity. Psychol. Aging 29:284. doi: 10.1037/a0036302

Ramsey, J. L., Neupert, S. D., Mroczek, D. K., and Spiro, A. III (2016). The effects of daily co-occurrence of affect on older adults' reactivity to health stressors. Psychol. Health 31, 364-378. doi: 10.1080/08870446. 2015.1111368

Ready, R. E., Carvalho, J. O., and Weinberger, M. I. (2008). Emotional complexity in younger, midlife, and older adults. Psychol. Aging 23:928. doi: 10.1037/ a0014003

Ready, R. E., Weinberger, M. I., and Jones, K. M. (2007). How happy have you felt lately? Two diary studies of emotion recall in older and younger adults. Cogn. Emot. 21, 728-757. doi: 10.1080/02699930600948269

Reich, J. W., Zautra, A. J., and Davis, M. (2003). Dimensions of affect relationships: models and their integrative implications. Rev. Gen. Psychol. 7, 66-83. doi: 10.1037/1089-2680.7.1.66

Roseman, I. J. (1984). Cognitive determinants of emotion: A structural theory. Rev. Pers. Soc. Psychol. 5, 11-36.

Rothman, N. B., Pratt, M. G., Rees, L., and Vogus, T. J. (2017). Understanding the dual nature of ambivalence: why and when ambivalence leads to good and bad outcomes. Acad. Manage. Ann. 11, 33-72. doi: 10.5465/ annals.2014.0066

Russell, J. A. (2003). Core affect and the psychological construction of emotion. Psychol. Rev. 110:145. doi: 10.1037/0033-295X.110.1.145
Russell, J. A. (2017). Mixed emotions viewed from the psychological constructionist perspective. Emot. Rev. 9, 111-117. doi: 10.1177/1754073916639658

Sarfraz, M., Qun, W., Abdullah, M. I., and Tahir, S. (2019). Authentic leadership, Hope, work engagement and creativity. J. Appl. Manage. Invest. 8, 19-31.

Scott, S. B., Sliwinski, M. J., Mogle, J. A., and Almeida, D. M. (2014). Age, stress, and emotional complexity: results from two studies of daily experiences. Psychol. Aging 29:577. doi: 10.1037/a0037282

Trudel-Fitzgerald, C., Qureshi, F., Appleton, A. A., and Kubzansky, L. D. (2017). A healthy mix of emotions: underlying biological pathways linking emotions to physical health. Curr. Opin. Behav. Sci. 15, 16-21. doi: 10.1016/j. cobeha.2017.05.003

Watson, D., Clark, L. A., and Tellegen, A. (1988). Development and validation of brief measures of positive and negative affect: the PANAS scales. J. Pers. Soc. Psychol. 54:1063. doi: 10.1037/0022-3514.54.6.1063

Wessman, A. E., and Ricks, D. F. (1966). Mood and Personality. New York: Holt, Rinehart and Winston.

Xiaolong, T., Gull, N., Asghar, M., and Jianmin, Z. (2021). The relationship between polychronicity and job-affective well-being: The moderator role of workplace incivility in healthcare staff. Work 70, 1267-1277. doi: 10.3233/ WOR-205286

Zautra, A. J., Affleck, G. G., Tennen, H., Reich, J. W., and Davis, M. C. (2005). Dynamic approaches to emotions and stress in everyday life: Bolger and Zuckerman reloaded with positive as well as negative affects. J. Pers. 73, 1511-1538. doi: 10.1111/j.0022-3506.2005.00357.x

Conflict of Interest: The authors declare that the research was conducted in the absence of any commercial or financial relationships that could be construed as a potential conflict of interest.

Publisher's Note: All claims expressed in this article are solely those of the authors and do not necessarily represent those of their affiliated organizations, or those of the publisher, the editors and the reviewers. Any product that may be evaluated in this article, or claim that may be made by its manufacturer, is not guaranteed or endorsed by the publisher.

Copyright (c) 2022 Dong and Xu. This is an open-access article distributed under the terms of the Creative Commons Attribution License (CC BY). The use, distribution or reproduction in other forums is permitted, provided the original author(s) and the copyright owner(s) are credited and that the original publication in this journal is cited, in accordance with accepted academic practice. No use, distribution or reproduction is permitted which does not comply with these terms. 